\title{
Growing Popularity of Ultrasensitive Microcalorimetry
}

\section{Eva Judy and Nand Kishore*}

Department of Chemistry, Indian Institute of Technology Bombay, Powai, Mumbai, India

*Corresponding author: Kishore N, Department of Chemistry, Indian Institute of Technology Bombay, Powai, Mumbai, India, Tel: 022-2576-7151; E-mail: nandk@chem.iitb.ac.in

Rec date: May 22, 2016, Acc date: Aug 04, 2016, Pub date: Aug 06, 2016

Copyright: (C) 2016 Judy E, et al. This is an open-access article distributed under the terms of the Creative Commons Attribution License, which permits unrestricted use, distribution, and reproduction in any medium, provided the original author and source are credited.

\section{Abstract}

Non covalent interactions in biological macromolecules mediate almost all the chemical processes in living systems. Developments in microcalorimetry has enabled a quantitative understanding of such interactions and contributed significantly to biotechnology, medicine, and novel drug design. This report addresses advancement and use of ultrasensitive microcalorimetry in connecting bioenergetics with possible applications in chemical and biological processes.

Keywords: Bioenergetics; Isothermal titration calorimetry; Differential scanning calorimetry; Novel drug design; Biothermodynamics; Protein folding and stability

\section{Introduction}

Most of the interactions which are essential for cellular organization or proper functioning of biological macromolecules are non-covalent in nature. A variety of ligands which can act as potential drugs, also interact via non-covalent interactions. Precise and accurate measurements of such weak interactions and correlation with structure and functional groups of reacting species can provide insights into functions and organization of such biologically important systems, and rational drug design. The conformational stability of a biological macromolecule is important for its function. The techniques which have rapidly grown in addressing these issues successfully are Isothermal Titration Calorimetry (ITC) and Differential Scanning Calorimetry (DSC).

Tronac titration calorimeter, prototype model of which was developed by Christensen and Izatt [1] in the year 1968 was mainly useful for the measurement of excess enthalpies or heats of dilutions. The sensitivity and amount of sample requirement did not allow this instrument to be a popular choice amongst scientists having biological interests. Efforts were continuously being put to develop calorimeters in which the reaction vessel was changed from an open to an adiabatic system with improved sensitivity. Such calorimeters either indigenous or commercially available were accurately able to determine the value of heat of neutralization of acid by alkali close to $-13.3 \mathrm{kcal}$ mol-1 which is amongst the best values known in literature [2]. However, the measurement of heats of interaction in biologically important reactions was not possible to high level of accuracy because of the above mentioned reasons. The differential scanning calorimeters started appearing in mid-sixties [3-5]. With the developments in technology, the sensitivities of ITC and DSC improved manifolds. Therefore, these techniques have become fundamental choice due to their inherent capability to determine thermodynamics of biologically important reactions irrespective of the limitations on molecular mass, intrinsic/ extrinsic labeling, immobilization of species or stringent requirement on transparency of the solutions. Currently, the ultrasensitive isothermal titration calorimeters which have made an impact in biologically important systems are VP-ITC and ITC200 from Malvern (Microcal), and Nano ITC Standard Volume and Nano ITC Low Volume from TA Instruments. Further developments in terms of improved sensitivity and low volume requirements are constantly being tried by these manufacturers (for example, recently launched PEAQ ITC by Malvern, and Affinity ITC by TA Instruments).

Differential Scanning Calorimetry has enabled understanding the forces which are responsible for the folded conformation of proteins and understanding protein folding intermediates. Not only just for monitoring the thermal stability of biological macromolecules, DSC is also extremely useful in unraveling the mechanism of protein unfolding. Reversible thermal unfolding transitions of proteins allow measurements of both calorimetric and Van't Hoffenthalpies, comparison of which suggests absence or presence of intermediate states, or onset of association of protein molecules during the thermal unfolding process $[6,7]$. Appearance of reduced thermal transitions or complete disappearance of cooperative transitions have been reported by different research groups for thermal unfolding of proteins from molten globule to unfolded state [8,9]. Nevertheless, whether it is reduced thermal transition with ratio of Van't Hoff to calorimetric enthalpy less than unity or disappearance of thermal transition, either non two-state unfolding mechanism or denatured state of the protein is inferred which can further be confirmed by using circular dichroism or fluorescence spectroscopy $[10,11]$. The role of specific amino acid residues in maintaining the three dimensional native conformation of proteins has also been answered by DSC through mutant proteins [12-14].

Desired affinity, selectivity, and solubility have been major challenges in discovery of novel drugs. The significance of enthalpic contribution in affinity by choosing examples of HIV protease inhibitors and statins from the first in class to the best in class over a period of about 12 years have been elegantly described by Ernesto Friere [15]. Though unplanned, the emergence of new drugs with stronger exothermic association as a key parameter indicates undisputed and unquestioned role of direct isothermal titration calorimetry in deriving guidelines for target oriented synthesis and rational drug design. Such a correlation can be established only if the energetics of interaction can be correlated with the functional groups both on the drug and at the binding site, suitable alterations of which 
Page 2 of 3

leads to improved affinity and selectivity. Exploding synthetic approaches to obtain potential novel drugs can attain focus with the availability of such guidelines.

The role of calorimetry and thermodynamics in biological macromolecules and drug design has been acknowledged from time to time [16-19]. ITC is also being employed in understanding the interactions of nucleic acids with variety of molecules ranging from small synthetic compounds to large proteins [20-22]. The result of such studies have great potential on design and evaluation of DNA interaction of small molecules that can enter cells and modify function of targeted cells in a programmed manner. Application of microcalorimetry has led to discovery of several potential compounds targeted at inhibition of telomerase enzyme which is an excellent method to selectively attack cancer cells $[23,24]$. Efforts directed towards discovery and characterization of small molecules that target the RaS-like GTPase, pathway components of which are currently being studied as breakthroughs for potential cancer therapies [25]. Clinical applications of Plasma thermograms have also been discussed [26]. Recently the applications of ITC in Nanoscience and Nanotechnology ranging from understanding agglomeration or stabilization mechanism of nanoparticles [27] to biomolecular nanoparticle interactions [28] have been reported or reviewed. Some other recently reported applications of ITC include monitoring the growth of bacterial activity which can be beneficial in designing antimicrobial agents [29]. Calorimetry has also found its use in the investigation of the fate of food products in the gastrointestinal tract [30]. Interaction of proteins with essential 1st row transition metals has also recently been reported [31,32]. Isothermal titration calorimetry and differential scanning calorimetry have also found role in getting insights into inhibition of aggregation or fibrillation of proteins, which is essential in prevention of neurodegenerative diseases [33,34].

Subsequent to the optimum design of the experiments and obtaining high quality data, suitable data analysis is extremely important. Even though, the data analysis software provided by instrument manufacturers is sufficient most of the times, situations may arise where additional modeling is required. Brautigam et al. [35] have recently suggested methods for global analysis of ITC calorimetric data for interaction of macromolecules.

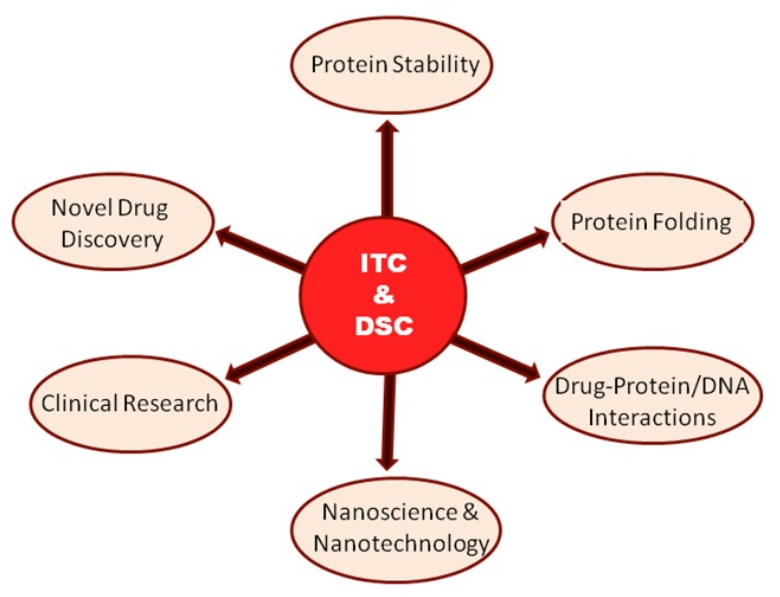

Figure 1: Potential applications of ITC and DSC.
Technical advances in isothermal titration calorimetry and differential scanning calorimetry will undoubtedly broaden the scope of their applications (Figure 1) in the years to come and make a huge impact in fundamental chemical and biological research, as well as in applied science and technology including those targeted at drug development, and finding answers to much awaited solution to protein folding problem.

\section{References}

1. Christensen JJ, JohnstonHD, IzattRM (1968) An isothermal titration calorimeter. Rev Sci Instrum 39: 1356-1359.

2. Parker VB (1965) National Standard Reference Data Series. National Bureau of Standards 2 (Category 5. Thermodynamic and Transport Properties).

3. Privalov PL, Monaselidze DR, Mrevilishvili GM, Magaldadze VA (1964) Intramolecular heat of fusion of macromolecules. Zh Eksp Teor Fiz (USSR) 47: 2073-2079.

4. Gill SJ, Beck K (1965) Differential heatapacity calorimeter for polymer transition studies. Rev Sci Instrum 36: 274-276.

5. Danford R, Kraukauer H, Sturtevant JM (1967) Differential calorimetry of thermally induced processes in solution. Rev Sci Instrum 38: 484-491.

6. Sturtevant JM (1987) Biochemical applications of differential scanning calorimetry. Ann Rev Phys Chem 38: 463-488.

7. Privalov PL, Khechinashvili NN (1974) A thermodynamic approach to the problem of stabilization of globular protein structure: a calorimetric study. J Mol Biol 86: 665-684.

8. Xie D, Bhakuni V, Freire E (1991) Calorimetric determination of the energetics of the molten globule intermediate in protein folding: apoalpha-lactalbumin. Biochemistry 30: 10673-10678.

9. Yutani K, Ogasahara K, Kuwajima K (1992) Absence of the thermal transition in apo-alpha-lactalbumin in the molten globule state. A study by differential scanning microcalorimetry. J Mol Biol 228: 347-350.

10. Xu Q, Keiderling TA (2004) Effect of sodium dodecyl sulfate on folding and thermal stability of acid-denatured cytochrome c: a spectroscopic approach. Protein Sci 13: 2949-2959.

11. Harder ME, Deinzer ML, Leid ME, Schimerlik MI (2004) Global analysis of three-state protein unfolding data. Protein Sci 13: 2207-2222.

12. Smith CK, Bu Z, Anderson KS, Sturtevant JM, Engelman DM, et al. (1996) Surface point mutations that significantly alter the structure and stability of a protein's denatured state. Protein Sci 5: 2009-2019.

13. Sturtevant JM, Yu MH, Haase-Pettingell C, King J (1989) Thermostability of temperature-sensitive folding mutants of the P22 tailspike protein. J Biol Chem 264: 10693-10698.

14. Ladbury JE, Kishore N, Hellinga HW, Wynn R, Sturtevant JM (1994) Thermodynamic effects of the reduction of the active-site disulfide of Escherichia coli thioredoxin explored by differential scanning calorimetry. Biochemistry 33: 3688-3692.

15. Freire E (2008) Do enthalpy and entropy distinguish first in class from best in class? Drug Discov Today 13: 869-874.

16. Chaires JB (2008) Calorimetry and thermodynamics in drug design. Annu Rev Biophys 37: 135-151.

17. Ladbury JE (2001) Isothermal titration calorimetry: application to structure-based drug design. Thermochim Acta 380: 209-215.

18. LadburyJE, DoyleML (2004) Biocalorimetry 2: Applications of calorimetry in the biological sciences. John Wiley and Sons, England.

19. Privalov PL, Dragan AI (2007) Microcalorimetry of biological macromolecules. Biophys Chem 126: 16-24.

20. Paul A, Chai Y, Boykin DW, Wilson WD (2015) Understanding mixed sequence DNA recognition by novel designed compounds: the kinetic and thermodynamic behaviour of azabenzimidazole diamidines. Biochemistry 54: 577-587. 
21. Ramos JP, Babu B, Chavda S, Liu Y, Plaunt A, et al. (2013) Affinity and kinetic modulation of polyamide-DNA interactions by N-modification of the heterocycles. Biopolymers 99: 497-507.

22. Freyer MW, Buscaglia R, Cashman D, Hyslop S, Wilson WD, et al. (2007) Binding of netropsin to several DNA constructs: Evidence for at least two different 1:1 complexes formed from an -AATT-containing ds-DNA construct and a single minor groove binding ligand. Biophys Chem 126: 186-196.

23. Prislan I, Lah J, Vesnaver G (2008) Diverse polymorphism of Gquadruplexes as a kinetic phenomenon. J Am Chem Soc 130: 14161-14169.

24. Arora A, Balasubramanian C, Kumar N, Agrawal S, Ojha RP, et al. (2008) Binding of berberine to human telomeric quadruplex - spectroscopic, calorimetric and molecular modeling studies. FEBS J 275: 3971-3983.

25. Yan C, Liu D, Li L, Wempe MF, Guin S, et al. (2014) Discovery and characterization of small molecules that target the GTPase Ral. Nature 515: 443-447.

26. Garbett NC, Mekmaysy CS, DeLeeuw L, Chaires JB (2015) Clinical application of plasma thermograms. Utility, practical approaches and considerations. Methods 76: 41-50.

27. Loosli F, Vitorazi L, Berret JF, Stoll S (2015) Isothermal titration calorimetry as a powerful tool to quantify and better understand agglomeration mechanisms during interaction processes between TiO2nanoparticles and humic acids. Environ Sci Nano 2: 541-550.
28. Huang R, Lau BLT (2016) Biomolecule-nanoparticle interactions: Elucidation of the thermodynamics by isothermal titration calorimetry. Biochim Biophys Acta 1860: 945-956.

29. Baldoni D, Hermann H, Frei R, Trampuz A, Steinhuber A (2009) Performance of microcalorimetry for early detection of methicillin resistance in clinical isolates of Staphylococcus aureus. J Clin Microbiol 47: 774-776.

30. Arroyo-Maya IJ, McClements DJ (2016) Application of ITC in foods: A powerful tool for understanding the gastrointestinal fate of lipophilic compounds. Biochim Biophys Acta 1860: 1026-1035.

31. Bou-Abdallah F, Giffune TR (2016) The thermodynamics of protein interactions with essential first row transition metals. Biochim Biophys Acta 1860: 879-891.

32. Terpstra T, McNally J, Han TH, Ha-Duong NT, El-Hage-Chahine JM, et al. (2014) Direct thermodynamic and kinetic measurements of $\mathrm{Fe}^{2+}$ and $\mathrm{Zn}^{2+}$ binding to human serum transferrin. J Inorg Biochem 136: 24-32.

33. Choudhary S, Kishore N (2014) Addressing mechanism of fibrillization/ aggregation and its prevention in presence of osmolytes: spectroscopic and calorimetric approach. Plos One 9: 1-10.

34. Choudhary S, Kishore N, Hosur RV (2015) Inhibition of insulin fibrillation by osmolytes: Mechanistic insights. Sci Rep 5: 17599.

35. Brautigam CA, Zhao H, Vargas C, Keller S, Schuck P (2016) Integration and global analysis of Isothermal titration calorimetry data for studying macromolecular interactions. Nat Protocols 11: 882-894. 